\title{
Postoperative Adjuvant Chemotherapy in Locally Advanced Cervical Cancer Patients with Optimal Response to Neoadjuvant Chemotherapy: A Retrospective Study
}

\section{Xiaojie Feng}

Zhengzhou University

Hongmin Chen

Zhengzhou University

Lei Li

Zhengzhou University

Ling Gao

Zhengzhou University

Xupeng Bai ( $\nabla$ xupeng.bai@gmail.com )

UNSW: University of New South Wales https://orcid.org/0000-0001-5393-1405

Li Wang

Zhengzhou University

Research article

Keywords: cervical cancer, neoadjuvant chemotherapy, optimal response, survival, toxicity

Posted Date: October 9th, 2020

DOI: https://doi.org/10.21203/rs.3.rs-88920/v1

License: (c) (i) This work is licensed under a Creative Commons Attribution 4.0 International License.

Read Full License 


\title{
Postoperative adjuvant chemotherapy in locally advanced cervical cancer patients with optimal response to neoadjuvant chemotherapy: a retrospective study
}

\author{
Xiaojie Feng ${ }^{1,2,3, *}$, Hongmin $\mathrm{Chen}^{1}$, Lei $\mathrm{Li}^{1}$, Ling Gao ${ }^{1}$, Xupeng Bai ${ }^{2,3,{ }^{*}}$ Li Wang ${ }^{1}$ \\ ${ }^{1}$ Department of Gynecologic Oncology, the Affiliated Cancer Hospital of Zhengzhou \\ University, Zhengzhou, Henan, China; \\ ${ }^{2}$ Cancer Care Centre, St George Hospital, Sydney, NSW, Australia; \\ ${ }^{3}$ St George and Sutherland Clinical School, Faculty of Medicine, UNSW Sydney, Sydney, \\ NSW, Australia.
}

\section{*Correspondence:}

Xiaojie Feng, Department of Gynecologic Oncology, the Affiliated Cancer Hospital of Zhengzhou University, Zhengzhou, Henan 450008, China. Tel/Fax: +86 37165587380 , E-mail: zlyyfengxiaojie1669@zzu.edu.cn

Xupeng Bai, Cancer Care Centre, St George Hospital, Kogarah, NSW 2217, Australia. Tel/Fax: +61 029113 1534; E-mail: xupeng.bai@gmail.com 


\section{Abstract}

Background. Few studies investigated the effectiveness of adjuvant chemotherapy (CT) in patients with optimal response to neoadjuvant CT (NACT), and an optimal number of treatment cycles for these patients remains unknown.

Methods. A total of 261 Chinese patients with FIGO stage Ib2-IIb cervical cancer who showed an optimal response to NACT were included after radical surgery (RS), and the disease-free survival (DFS) and overall survival (OS) of patients treated with different cycles of postoperative adjuvant CT were compared.

Results. We found that patients treated with different cycles of postoperative adjuvant CT were significantly better than those without further therapy. The multivariate analysis showed that postoperative adjuvant CT was an independent prognostic factor for DFS. However, there was no significant difference in the DFS and OS between patients who had 3 cycles of adjuvant CT and those with 6 cycles. Further analysis revealed a significant association of 6 cycles of adjuvant $\mathrm{CT}$ with increased risk of leukopenia, nausea/vomiting, and rash.

Conclusion. These data suggest that additional 3 cycles of adjuvant CT after NACT + RS may improve the clinical outcome of optimal responders in terms of DFS, OS, and drug toxicity.

Keywords: cervical cancer, neoadjuvant chemotherapy, optimal response, survival, toxicity 


\section{Background}

Cervical cancer is the second most common gynecologic cancer in developing countries. The estimated annual incidence and mortality of cervical cancer showed an income-dependent distribution pattern with the greatest disease burden in sub-Saharan Africa, Southeast Asia, and Latin America due to the poor access to screening and treatment [1]. Almost 530,000 new cases are diagnosed worldwide each year, and approximately 270,000 women die of cervical cancer annually [2].

Neoadjuvant chemotherapy (NACT) followed by radical surgery (RS) is considered as a valid alternative for locally advanced cervical cancer and utilized in many countries. The sequence contributes to a substantial improvement towards disease control and survival for stage Ib2-IIb tumors as compared to the radiotherapy (RT) alone [3]. Unfortunately, there were still around $20 \%-30 \%$ of patients treated with NACT + RS suffering from pelvic and/or extra-pelvic recurrence in 5 years, and long-term overall survival (OS) is still unsatisfactory [4]. For these reasons, postoperative adjuvant chemotherapy (CT) has been added with the intention of radical cure [5]. It was reported that additional 3-6 cycles of platinum-based adjuvant CT were of benefit for patients after NACT + RS in terms of 5-year OS and disease-free survival (DFS) [6-10].

However, few studies investigated the effectiveness of postoperative adjuvant CT in patients with optimal response to NACT, and an optimal number of treatment cycles for these patients remains unknown.

Therefore, this study aims to evaluate the effectiveness of postoperative adjuvant CT in 
cervical cancer patients with optimal response to NACT. Also, 3 and 6 cycles of adjuvant CT were compared in terms of OS, DFS, and drug toxicity. These data may provide clinical evidence for a better practice of adjuvant $\mathrm{CT}$ in cervical cancer patients.

\section{Methods}

\section{Study population}

This is a single-institution retrospective study, which was approved by the Medical Ethics Committee in the Affiliated Cancer Hospital of Zhengzhou University (Approval No.2017416) in accordance with the 1964 Helsinki declaration and its later amendments and with the Chinese laws and regulations. Informed consent is not required for the study. Between April 2009 and September 2016, patients with FIGO stage Ib2-IIb cervical cancer referred to the Department of Gynecologic Oncology were included in this study. The patient inclusion criteria included: 1) female with squamous cell, adenosquamous or adenocarcinoma of the cervix; 2) age between 18 and 75 years; 3) having normal hepatorenal, cardiac, and respiratory functions. The exclusion criteria included: 1) severe other organ injuries; 2) a history of any disease that may exert influence on this study and other unacceptable diseases, including abnormal bone marrow function, uncontrolled infection, diabetes mellitus, acquired immune deficiency syndrome, substance dependence, and neurological or mental diseases; 3) pregnancy. 


\section{Adjuvant chemotherapy}

All patients included in the study received NACT that is comprised of 2 cycles of the scheme cisplatin $75 \mathrm{mg} / \mathrm{m}^{2}$ and paclitaxel $175 \mathrm{mg} / \mathrm{m}^{2}$ at 3-week intervals. Then, patients were assessed to determine the treatment response according to the WHO criteria (WHO handbook for reporting results of cancer treatment: http://www.who.int/iris/handle /10665/37200). Patients with stable or progressive disease were not suitable for surgery, and thus they were excluded from this study and sent to radiotherapy.

Included patients underwent bilateral systematic pelvic lymph node dissection, Piver type II or III Piver-Rutledge radical hysterectomy, and bilateral salpingo-oophorectomy. Aortic lymphadenectomy was performed in patients with pelvic node disease or bulky aortic nodes.

After surgery, the pathological evaluation of surgical specimens was performed to further determine the response to NACT as a golden standard method. Patients who had positive nodes, positive surgical margins, or vaginal margins less than $0.5 \mathrm{~mm}$ were excluded from the study and submitted to RT. Complete response was defined as the complete disappearance of the tumor in the cervix with negative nodes. Optimal partial response was defined as a persistent residual disease with $<3 \mathrm{~mm}$ stromal invasion, including in situ carcinoma on the surgical specimen and negative lymphatic metastasis. Overall optimal response was defined as the sum of complete and optimal partial response. Patients who did not achieve optimal response were excluded from this study.

Thereafter, patients received 3 to 6 cycles of adjuvant chemotherapy at 3-week intervals beginning 3-4 weeks after surgery according to the same regime with NACT. 
Chemotherapeutic toxicity was recorded and graded according to the WHO criteria (WHO handbook for reporting results of cancer treatment: http://www.who.int/iris/handle /10665/37200). Treatment was delayed, or dosage was decreased in the case of G3 toxicity. Treatment was discontinued in the case of G4 or life-threatening toxicity. Cisplatin was replaced with carboplatin and paclitaxel was replaced with topotecan if severe hypersensitivity reaction occurred.

\section{Follow-up surveys}

Real-time demographic and clinical data were obtained from the electronic medical record system at the Department of Gynecologic Oncology. At the end of adjuvant CT, all patients were followed up every 3-4 months for the first 2 years, then every 6 months for the following 3 years, and annually thereafter. Each follow-up included an essential interview, physical examination, and vaginal cytology for identifying lower genital tract tumors. Computed tomography or magnetic resonance imaging was used to examine abdominal and pelvic regions. The biopsy was performed to evaluate suspicious cases of recurrent cancer. Cancer recurrence evidence was defined as either regional recurrence or distant metastasis.

\section{Statistical analysis}

DFS was calculated from the date of the first cycle of NACT to the date of documented evidence of recurrence, while OS was defined as the time from the first cycle of NACT to 
death or last follow-up. Survival curves were generated based on the Kaplan-Meier method and compared using the log-rank test. Cox proportional hazard model was employed for multivariate analysis. Other analyses were done using the chi-square test and Fisher test. Statistical significance was set at $P<0.05$. All computations were performed using SPSS version 23.0 (IBM Analysis, USA).

\section{Results}

Effect of postoperative adjuvant CT on DFS and OS in patients with optimal response to $N A C T$

From April 2009 to September 2016, 4373 patients were assessed according to the inclusion and exclusion criteria. 1654 patients received NACT + RS. Thereinto, 261 (15.8\%) patients who achieved an optimal response to NACT were included in the analysis. The pathological response was complete in 61 patients, optimal partial in 200. $152(58.2 \%)$ patients underwent type III Piver-Rutledge radical hysterectomy, while 109 (41.8\%) patients underwent type II Piver-Rutledge radical hysterectomy.

After surgery, $60(23.0 \%)$ patients were not treated with further adjuvant CT; $101(38.7 \%)$ patients received 3 cycles of adjuvant CT; $16(6.1 \%)$ patients underwent 4 cycles of adjuvant CT; $22(8.4 \%)$ patients had 5 cycles of adjuvant CT; $62(23.8 \%)$ patients underwent 6 cycles of adjuvant CT. There are $15(5.7 \%)$ and $6(2.3 \%)$ patients showing hypersensitivity reactions to Cisplatin and Paclitaxel, respectively, and thus the drugs were replaced as described in the 
method.

At the time of the analysis, $17(6.5 \%)$ out of the 261 patients relapsed after a median time of 15.8 months (range, 5.5 to 43.3 months); $8(3.0 \%)$ out of the 261 patients died after a median time of 22.9 months (range, 19.3 to 41.3 months). As shown in Table 1, the recurrent disease was pelvic in $8(3.0 \%)$ cases, extra-pelvic (aortic or distant) in $6(2.3 \%)$ cases, and both pelvic and extra-pelvic in $2(0.8 \%)$ cases. According to the cycle of adjuvant CT, tumor relapsed in $8(13.3 \%)$ out of the 60 patients without further therapy [5 pelvic recurrences $(2$ died), and 2 extrapelvic recurrences (died), and 1 pelvic + extrapelvic recurrence (died)], 5 $(5.0 \%)$ out of the 101 patients with 3 cycles [ 2 pelvic recurrences, 2 extra-pelvic recurrences ( 1 died), and 1 pelvic + extra-pelvic recurrence (died)], $1(6.3 \%)$ out of the 16 patients with 4 cycles [1 pelvic recurrence (died)], $1(4.5 \%)$ out of the 22 patients with 5 cycles (1 extra-pelvic recurrence), and $2(3.2 \%)$ out of the 62 patients with 6 cycles [1 pelvic recurrence and 1 extra-pelvic recurrences (died)] (Table 1).

DFS and OS of patients treated with postoperative adjuvant CT were significantly better as compared to patients without further therapy $(P=0.012, \mathrm{HR}=3.162,95 \% \mathrm{CI}=$ 1.002-5.971 and $P=0.047, \mathrm{HR}=3.207,95 \% \mathrm{CI}=1.080-7.782$, respectively) (Fig. $1 \mathrm{~A}$ and $\mathrm{B}$ ).

Multivariate analysis on the whole series using Cox proportional hazard model showed that DFS and OS rate were significantly higher in patients with squamous cell cancer than in 
those with adenosquamous $(P=0.0007, \mathrm{HR}=1.834,95 \% \mathrm{CI}=1.285-2.322$ and $P=0.0002$, $\mathrm{HR}=2.057,95 \% \mathrm{CI}=1.182-2.937$, respectively) (Table 2). Further analysis found that DFS was better in patients who received postoperative adjuvant $\mathrm{CT}$ than in those who $\operatorname{did}$ not $(P=$ $0.033, \mathrm{HR}=1.740,95 \% \mathrm{CI}=1.103-2.369)$, while the difference of OS between the two groups became nonsignificant after multivariate correction $(P=0.097)$ (Table 2).

Comparison of 3 and 6 cycles of postoperative adjuvant chemotherapy in patients with an optimal response in terms of DFS, OS, and toxicity

Next, patients with 3 or 6 cycles of adjuvant CT were selected for the following analysis to investigate the effect of the chemotherapeutic cycle on the prognosis of patients with an optimal response. The potential variables that may affect the chemotherapeutic cycle in the present study were assessed by univariate analysis, and there was no statistically significant difference between the two groups (Table 3).

There was no significant difference on the DFS and OS between patients who had 3 cycles of adjuvant $\mathrm{CT}$ and those who received 6 cycles $(P=0.618$ and $P=0.852$, respectively) (Fig. 2A and B).

The toxicity profile among these patients is shown in Table 4. The most frequent adverse reaction was leukopenia and then nausea/vomiting. Specifically, leukopenia occurred in $67.7 \%$ of patients with 6 cycles of $\mathrm{CT}$ and in $39.7 \%$ of patients with 3 cycles; nausea/vomiting occurred in $59.7 \%$ of patients with 6 cycles of CT and in $36.6 \%$ of patients with 3 cycles. Analysis of these data showed statistically significant differences between the 
two groups in terms of leukopenia $(P=0.003, \mathrm{RR}=2.683,95 \% \mathrm{CI}=1.200-5.998)$ and nausea/vomiting $(P=0.017, \mathrm{RR}=1.314,95 \% \mathrm{CI}=1.176-2.222)$. Furthermore, the rash incidence also showed a statistically significant difference between the two groups $(P=0.023$, $\mathrm{RR}=3.889,95 \% \mathrm{CI}=1.073-14.097)$. In contrast, there were no significant differences on thrombocytopenia $(P=0.413)$, anemia $(P=0.505)$, absolute neutrophil $(P=1.000)$, peripheral sensory neuropathy $(P=0.329)$, and hepatotoxicity/Nephrotoxicity $(P=1.000)$ between the two groups.

\section{Discussion}

The clinical practice of postoperative adjuvant CT for locally advanced cervical cancer patients with optimal response to NACT seems to be less verified. This is because no pathological risk factor can be used to evaluate the risk of recurrence and death in these optimal responders, and no standard guideline can be referred to decide the optimized course of adjuvant CT. Many studies reported that the survival time of optimal pathological responders was significantly longer than that of non-optimal responders, considering optimal response as a favorable prognostic factor and surrogate endpoint for survival $[4,5,11,12]$, which may explain why the postoperative management for these patients did not gain enough attention.

In fact, although most optimal responders survived without evidence of recurrence for a short time, the long-term recurrent cases did exist in them. In the current study, the overall 
recurrence and death rates were approximately $6.5 \%$ and $3.1 \%$, respectively. In fact, a small minority of optimal responders carried invisible distant micro-metastasis that might explain the tumor relapse after several years [13]. Compared with adjuvant RT that shows an effective local recurrence control, adjuvant CT has an advantage in treating extra-pelvic metastasis [14]. Consistently, $5.0 \%$ of patients (3 out of 60 ) without therapy experienced extra-pelvic recurrence as compared to $3.0 \%$ of patients with adjuvant CT (6 out of 201) in our study, suggestive of the importance of additional adjuvant CT in eliminating the potential metastatic lesions. More importantly, patients with adjuvant CT showed higher DFS and OS rate as compared to those without further therapy, indicating that adjuvant CT can still improve the prognosis even if they obtained an optimal response to NACT. F Landoni, et al. [13] reported that there was no significant difference on the risk of recurrence between 0 and 2 cycles of adjuvant CT in overall optimal responders (11.1\% vs. 11.1\%). It is complicated to compare them to our results because the sample size and adjuvant CT cycle they used are less than ours. However, we noticed that the recurrence rate $(13.3 \%)$ in patients without therapy in our study is higher than European studies (range 10.9\%-11.1\%) [4, 13]. The possible explanation is the different NACT cycle because in Asian countries, such as China and Japan, 2 cycles of platinum-based NACT were widely accepted for patients [11, 12, 15-17], whereas 3 cycles were widely used in European countries [4, 7, 18].

In addition, we observed that the subtype of cancer in most optimal responders was squamous cell types, suggesting squamous cell type cervical cancer are more sensitive towards CT than adenosquamous cancer and adenocarcinoma. This is consistent with a study 
indicating that the chemotherapeutic response was more favorable in squamous cell carcinomas $(87 \%)$ than that in adenocarcinomas $(38 \%)(P=0.01)$ [19]. Of note, no patient with stage IIb was found to achieve optimal response in the current study because of the finding of high-risk and/or intermediate risk factors after surgery, such as positive nodes and deep muscle invasion.

Compared with RT, CT is a first-line adjuvant treatment for optimal responders in case of recurrent disease because RT cannot be used repeatedly. Some studies indicated that the adjuvant $\mathrm{CT}$ could be given according to the number of high-risk and/or intermediate-risk factors that patients have $[6,20]$. However, no risk or reliable prognostic factor was identified in optimal responders after surgery for choosing the optimized CT cycle. As a result, the duration of adjuvant $\mathrm{CT}$ for these patients was performed according to the institutional experience, ranging from 0 to 6 cycles. In our experience, 3-6 cycles of CT were usually used for patients based on individual tolerance. In this study, we found that adjuvant CT was an independent prognostic factor for optimal responders, whereas there was no significant difference on the DFS and OS between 3 and 6 cycles.

On the other hand, 3 cycles of CT showed a favorable toxicity profile as compared to 6 cycles. A significantly higher incidence of leukopenia and nausea/vomiting was observed in the 6-cycle group. Our data of hematological toxicity are comparable to other studies regarding patients submitted to 6 courses of platinum-based adjuvant CT which showed $9.2 \%$ thrombocytopenia, $23.2 \%$ anemia, $15.6 \%$ febrile neutropenia, whereas the incidence of nausea/vomiting and neurotoxicity in our study are much higher than other studies showing 
$17.9 \%$ vomiting and $3.3 \%$ neurotoxicity [21]. The possible explanation for this might be the racial difference. In addition, 6 cycles of $\mathrm{CT}$ also induced an increase in the incidence of G2 rash. These data suggest that 3 courses of adjuvant CT are more tolerated with acceptable adverse reactions, which may be suitable for patients to both comply with the following therapy and improve survival quality.

\section{Conclusions}

The findings from this study indicates that additional cycles of adjuvant CT after NACT + radical surgery may improve the clinical outcome of optimal responders. Compared with 6 cycles, 3 cycles of adjuvant CT could be of benefit for these patients in terms of DFS, OS, and drug toxicity.

\section{Declarations}

\section{Ethics approval and consent to participate}

This is a single-institution retrospective study, which was approved by the Medical Ethics Committee in the Affiliated Cancer Hospital of Zhengzhou University (Approval No.2017416) in accordance with the 1964 Helsinki declaration and its later amendments and with the Chinese laws and regulations. Informed consent is not required for the study. 


\section{Consent for publication}

Not applicable.

\section{Availability of data and materials}

The datasets used and/or analyzed during the current study are available from the corresponding author on reasonable request.

\section{Competing interest}

All authors declare that there is no any conflict of interest.

\section{Funding}

This study was supported by the Project of Science and Technology Department of Henan Province [192102310066]. The authors would also like to acknowledge the funding support from UNSW Sydney and China Scholarship Council. 


\section{Author contributions}

X.F. conceived and design the study. X.F. performed literature research and clinical data

collection. H.C., L.L., L.G., and L.W. assisted with the study. X.F. and X.B. performed data analysis and manuscript preparation.

\section{Acknowledgments}

Not applicable.

\section{References}

1. Torre LA, Bray F, Siegel RL, Ferlay J, Lortet-Tieulent J, Jemal A: Global cancer statistics, 2012. $C A$ : a cancer journal for clinicians 2015, 65(2):87-108.

2. Arbyn M, Castellsague X, de Sanjose S, Bruni L, Saraiya M, Bray F, Ferlay J: Worldwide burden of cervical cancer in 2008. Annals of oncology : official journal of the European Society for Medical Oncology 2011, 22(12):2675-2686.

3. Benedetti-Panici P, Greggi S, Colombo A, Amoroso M, Smaniotto D, Giannarelli D, ... Landoni F: Neoadjuvant chemotherapy and radical surgery versus exclusive radiotherapy in locally advanced squamous cell cervical cancer: results from the Italian multicenter randomized study. Journal of clinical oncology : official journal of the American Society of Clinical Oncology 2002, 20(1):179-188.

4. Gadducci A, Sartori E, Maggino T, Zola P, Cosio S, Zizioli V, . . . Landoni F: Pathological response on surgical samples is an independent prognostic variable for patients with Stage Ib2-IIb cervical cancer treated with neoadjuvant chemotherapy and radical hysterectomy: an Italian multicenter retrospective study (CTF Study). Gynecologic oncology 2013, 131(3):640-644.

5. Buda A, Lissoni AA, Floriani I, Biagioli E, Gerardi C, Bonazzi C, . . Milani R: Long-Term Clinical Benefits of Neoadjuvant Chemotherapy in Women With Locally Advanced Cervical Cancer: 
Validity of Pathological Response as Surrogate Endpoint of Survival. Int J Gynecol Cancer 2015, 25(8):1468-1475.

6. Takeshima N, Umayahara K, Fujiwara K, Hirai Y, Takizawa K, Hasumi K: Treatment results of adjuvant chemotherapy after radical hysterectomy for intermediate- and high-risk stage IB-IIA cervical cancer. Gynecologic oncology 2006, 103(2):618-622.

7. Angioli R, Plotti F, Montera R, Aloisi A, Luvero D, Capriglione S, . . Benedetti-Panici P: Neoadjuvant chemotherapy plus radical surgery followed by chemotherapy in locally advanced cervical cancer. Gynecologic oncology 2012, 127(2):290-296.

8. Hosaka M, Watari H, Kato T, Odagiri T, Konno Y, Endo D, . . . Sakuragi N: Clinical efficacy of paclitaxel/cisplatin as an adjuvant chemotherapy for patients with cervical cancer who underwent radical hysterectomy and systematic lymphadenectomy. J Surg Oncol 2012, 105(6):612-616.

9. Li S, Hu T, Chen Y, Zhou H, Li X, Cheng X, . . Ma D: Adjuvant chemotherapy, a valuable alternative option in selected patients with cervical cancer. PloS one 2013, 8(9):e73837.

10. Angioli R, Plotti F, Aloisi A, Scaletta G, Capriglione S, Luvero D, . . Panici PB: A randomized controlled trial comparing four versus six courses of adjuvant platinum-based chemotherapy in locally advanced cervical cancer patients previously treated with neo-adjuvant chemotherapy plus radical surgery. Gynecologic oncology 2015, 139(3):433-438.

11. Huang K, Sun H, Chen Z, Li X, Wang S, Zhao X, . . Wang S: Optimal pathological response indicated better long-term outcome among patients with stage IB2 to IIB cervical cancer submitted to neoadjuvant chemotherapy. Scientific reports 2016, 6:28278.

12. Liang Y, Lu B, Chen X, Qin J, Cheng X, Xie X, Lu W: Prognostic value of pathological response to neoadjuvant chemotherapy in bulky stage Ib2 and IIa cervical squamous cell cancer patients. Virchows Archiv : an international journal of pathology 2016, 468(3):329-336.

13. Landoni F, Sartori E, Maggino T, Zola P, Zanagnolo V, Cosio S, . . Gadducci A: Is there a role for postoperative treatment in patients with stage Ib2-IIb cervical cancer treated with neo-adjuvant chemotherapy and radical surgery? An Italian multicenter retrospective study. Gynecologic oncology 2014, 132(3):611-617.

14. Lahousen M, Haas J, Pickel H, Hackl A, Kurz C, Ogris H, . . Winter R: Chemotherapy versus radiotherapy versus observation for high-risk cervical carcinoma after radical hysterectomy: A randomized, prospective, multicenter trial. Gynecologic oncology 1999, 73(2):196-201.

15. Shu T, Zhao D, Li B, Wang Y, Liu S, Li P, ... Wu L: Prognostic evaluation of postoperative adjuvant therapy for operable cervical cancer: 10 years' experience of National Cancer Center in China. Chin J Cancer Res 2017, 29(6):510-520.

16. Shoji T, Takatori E, Furutake Y, Takada A, Nagasawa T, Omi H, . . . Sugiyama T: Phase II clinical study of neoadjuvant chemotherapy with CDDP/CPT-11 regimen in combination with radical 
hysterectomy for cervical cancer with a bulky mass. International journal of clinical oncology 2016, 21(6):1120-1127.

17. Takatori E, Shoji T, Omi H, Kagabu M, Miura F, Takeuchi S, . . Sugiyama T: Analysis of prognostic factors for patients with bulky squamous cell carcinoma of the uterine cervix who underwent neoadjuvant chemotherapy followed by radical hysterectomy. International journal of clinical oncology 2015, 20(2):345-350.

18. Buda A, Fossati R, Colombo N, Fei F, Floriani I, Gueli Alletti D, . . Mangioni C: Randomized trial of neoadjuvant chemotherapy comparing paclitaxel, ifosfamide, and cisplatin with ifosfamide and cisplatin followed by radical surgery in patients with locally advanced squamous cell cervical carcinoma: the SNAP01 (Studio Neo-Adjuvante Portio) Italian Collaborative Study. Journal of clinical oncology : official journal of the American Society of Clinical Oncology 2005, 23(18):4137-4145.

19. Namkoong SE, Park JS, Kim JW, Bae SN, Han GT, Lee JM, . . Kim SJ: Comparative study of the patients with locally advanced stages I and II cervical cancer treated by radical surgery with and without preoperative adjuvant chemotherapy. Gynecologic oncology 1995, 59(1):136-142.

20. Li R, Lu ST, Si JG, Liu B, Wang H, Mei YY, Linghu H: Prognostic value of responsiveness of neoadjuvant chemotherapy before surgery for patients with stage IB(2)/IIA(2) cervical cancer. Gynecologic oncology 2013, 128(3):524-529.

21. Lorusso D, Petrelli F, Coinu A, Raspagliesi F, Barni S: A systematic review comparing cisplatin and carboplatin plus paclitaxel-based chemotherapy for recurrent or metastatic cervical cancer. Gynecologic oncology 2014, 133(1):117-123. 


\section{Figure legends}

A

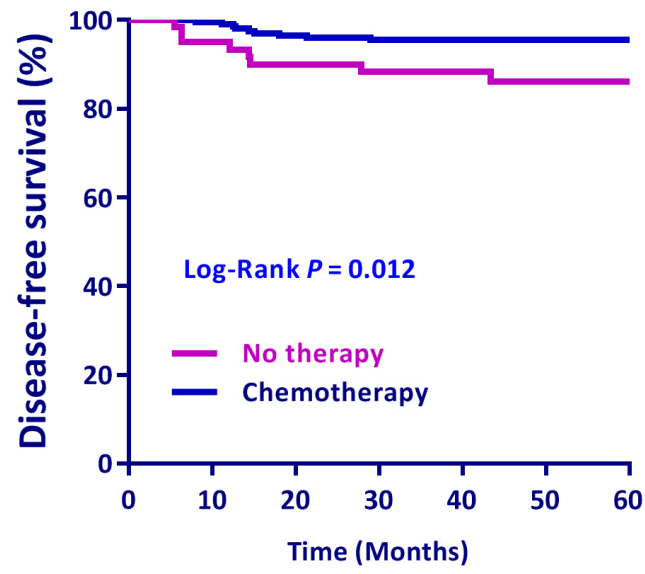

B

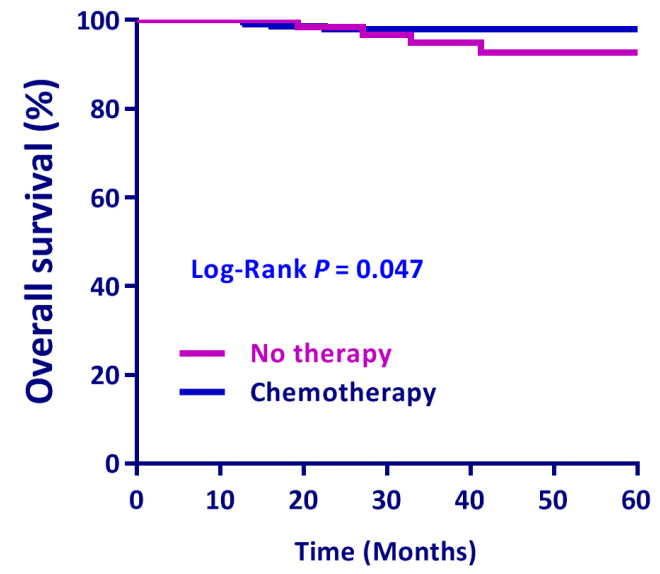

Figure 1. (A) Disease-free survival (DFS) and (B) overall survival (OS) by postoperative chemotherapy. Differences in DFS and OS by treatment were evaluated using the log-rank test. $P<0.05$ was statistically significant.

A

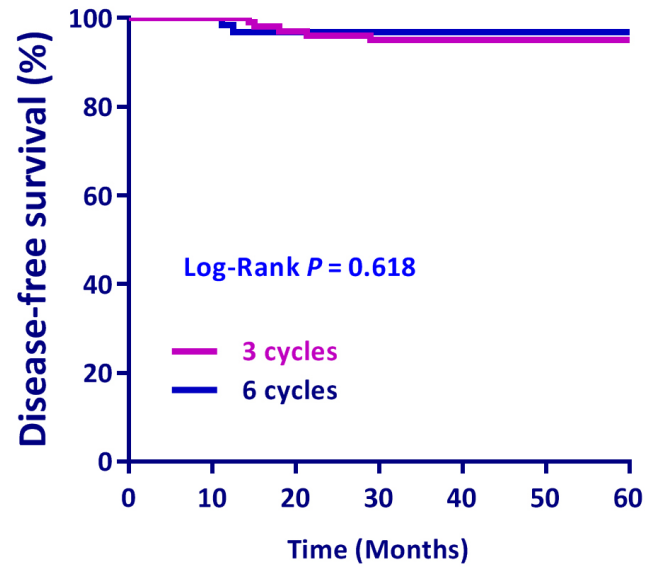

B

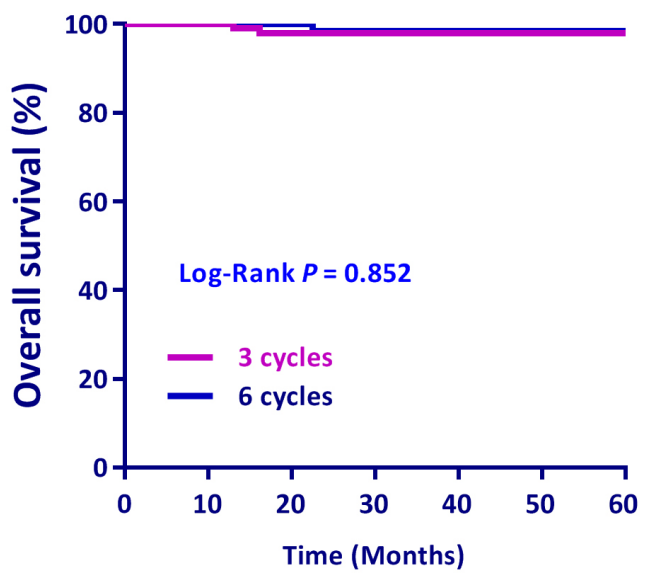

Figure 2. (A) Disease-free survival (DFS) and (B) overall survival (OS) by postoperative chemotherapeutic cycle. Differences in DFS and OS by treatment were evaluated using the log-rank test. 
Table 1. Recurrence and death rates in patients with CR according to the postoperative adjuvant chemotherapeutic cycle.

\begin{tabular}{ccccccc}
\hline \multirow{2}{*}{$\begin{array}{c}\text { Adjuvant } \\
\text { chemotherapy }\end{array}$} & Pts & \multicolumn{3}{c}{ Recurrence and death profile } & \multicolumn{2}{c}{ Overall } \\
\cline { 3 - 7 } & & $\mathbf{P}$ & $\mathbf{E X}$ & $\mathbf{P}+\mathbf{E X}$ & Recurrence & Death \\
\hline 0 cycles & 60 & $5(2$ died $)$ & 2 (died) & 1 (died) & $8(13.3 \%)$ & $4(6.7 \%)$ \\
3 cycles & 101 & 2 & $2(1$ died $)$ & $1($ died $)$ & $5(5.0 \%)$ & $2(2.0 \%)$ \\
4 cycles & 16 & 0 & 1 (died) & 0 & $1(6.3 \%)$ & $1(6.3 \%)$ \\
5 cycles & 22 & 0 & 1 & 0 & $1(4.5 \%)$ & $0(0.0 \%)$ \\
6 cycles & 62 & 1 & 1 (died) & 0 & $2(3.2 \%)$ & $1(1.6 \%)$ \\
\hline
\end{tabular}

Abbreviations: CR, complete response; P, pelvic; EX, extra-pelvic. 
Table 2. Multivariate analysis of variables predictive of DFS and OS in patients with CR.

\begin{tabular}{|c|c|c|c|c|c|}
\hline Variables & Pts & 5-year DFS & $P$ value & 5-year OS & $P$-value \\
\hline \multicolumn{6}{|l|}{ Age } \\
\hline$\leq 46$ & 158 & $93.7 \%$ & 0.886 & $96.8 \%$ & 0.896 \\
\hline$>46$ & 103 & $91.9 \%$ & & $95.1 \%$ & \\
\hline \multicolumn{6}{|l|}{ Histology } \\
\hline Squamous cell & 258 & $93.5 \%$ & $0.0007^{*}$ & $96.8 \%$ & $0.0002 *$ \\
\hline Adenosquamous & 3 & $33.3 \%$ & & $33.3 \%$ & \\
\hline Adenocarcinoma & 0 & - & & - & \\
\hline \multicolumn{6}{|l|}{ FIGO stage } \\
\hline $\mathrm{Ib} 2$ & 177 & $93.2 \%$ & 0.834 & $97.2 \%$ & 0.745 \\
\hline IIa & 84 & $91.6 \%$ & & $93.0 \%$ & \\
\hline $\mathrm{IIb}$ & 0 & - & & - & \\
\hline \multicolumn{6}{|l|}{ Grade } \\
\hline G1 & 54 & $94.4 \%$ & 0.769 & $96.3 \%$ & 0.906 \\
\hline G2 & 68 & $94.1 \%$ & & $95.5 \%$ & \\
\hline G3 & 139 & $91.4 \%$ & & $96.4 \%$ & \\
\hline \multicolumn{6}{|l|}{ Adjuvant } \\
\hline \multicolumn{6}{|l|}{ chemotherapy } \\
\hline Yes & 201 & $95.5 \%$ & $0.033^{*}$ & $98.0 \%$ & 0.097 \\
\hline No & 60 & $86.1 \%$ & & $92.7 \%$ & \\
\hline
\end{tabular}

Abbreviations: DFS, disease-free survival; OS, overall survival, CR, complete response; Pts, patients; FIGO, International Federation of Gynecology and Obstetrics.

$* P<0.05$ was statistically significant. 
Table 3. Characteristics of patients treated with 3 or 6 cycles of adjuvant chemotherapy.

\begin{tabular}{llll}
\hline Variables & $\begin{array}{l}\mathbf{3} \text { cycles } \\
(\mathbf{n}=\mathbf{1 0 1})\end{array}$ & $\begin{array}{l}\mathbf{6} \text { cycles } \\
(\mathbf{n}=\mathbf{6 2})\end{array}$ & $\boldsymbol{P}$ value \\
\hline Age & & & \\
$\leq 46$ & $56(55.4 \%)$ & $25(59.7 \%)$ & 0.628 \\
$>46$ & $45(44.6 \%)$ & $25(40.3 \%)$ & \\
Histology & & & \\
Squamous cell & $101(100.0 \%)$ & $61(98.4 \%)$ & 0.380 \\
Adenosquamous & $0(0.0 \%)$ & $1(1.6 \%)$ & \\
FIGO stage & & & \\
Ib2 & $64(63.4 \%)$ & $40(64.5 \%)$ & 1.000 \\
IIa & $37(36.6 \%)$ & $22(35.5 \%)$ & \\
Grade & & & \\
G1 & $14(13.9 \%)$ & $9(14.5 \%)$ & \\
G2 & $37(36.6 \%)$ & $21(33.9 \%)$ & \\
G3 & $50(49.5 \%)$ & $32(51.6 \%)$ & \\
\hline
\end{tabular}

Abbreviations: FIGO, International Federation of Gynecology and Obstetrics. 
Table 4. The associations of adjuvant chemotherapy with toxicity in patients with CR.

\begin{tabular}{|c|c|c|c|}
\hline Toxicity & $\begin{array}{l}3 \text { cycles } \\
(n=101)\end{array}$ & $\begin{array}{l}6 \text { cycles } \\
(n=62)\end{array}$ & $P$ value \\
\hline \multicolumn{4}{|c|}{ Leukopenia } \\
\hline G3 & $35(34.7 \%)$ & $24(38.7 \%)$ & $0.003^{*}$ \\
\hline G4 & $5(5.0 \%)$ & $18(29.0 \%)$ & \\
\hline \multicolumn{4}{|c|}{ Thrombocytopenia } \\
\hline G3 & $7(6.9 \%)$ & $4(6.5 \%)$ & 0.413 \\
\hline G4 & $5(5.0 \%)$ & $8(12.9 \%)$ & \\
\hline \multicolumn{4}{|l|}{ Anemia } \\
\hline G3 & $11(10.9 \%)$ & $7(11.3 \%)$ & 0.505 \\
\hline G4 & $8(7.9 \%)$ & $10(16.1 \%)$ & \\
\hline \multicolumn{4}{|c|}{ Absolute neutrophil } \\
\hline G3 & $3(3.0 \%)$ & $10(16.1 \%)$ & 1.000 \\
\hline G4 & $1(1.0 \%)$ & $2(3.2 \%)$ & \\
\hline \multicolumn{4}{|c|}{ Nausea and vomiting } \\
\hline G3 & $28(27.7 \%)$ & $17(27.4 \%)$ & $0.017 *$ \\
\hline G4 & $9(8.9 \%)$ & $20(32.3 \%)$ & \\
\hline \multicolumn{4}{|c|}{ Peripheral sensory neuropathy } \\
\hline G3 & $2(2.0 \%)$ & $7(11.3 \%)$ & 0.329 \\
\hline G4 & $3(3.0 \%)$ & $3(4.8 \%)$ & \\
\hline \multicolumn{4}{|c|}{ Hepatotoxicity/Nephrotoxicity ${ }^{\mathrm{a}}$} \\
\hline G1 & $2(2.0 \%)$ & $4(6.5 \%)$ & 1.000 \\
\hline G2 & $1(1.0 \%)$ & $3(4.8 \%)$ & \\
\hline \multicolumn{4}{|c|}{ Dermatology (rash) ${ }^{\mathrm{a}}$} \\
\hline G1 & $7(6.9 \%)$ & $2(3.2 \%)$ & $0.023 *$ \\
\hline G2 & $2(2.0 \%)$ & $8(12.9 \%)$ & \\
\hline
\end{tabular}

Abbreviations: CR, complete response.

${ }^{\text {aNo }}$ G3-4 toxicity case was reported.

$* P<0.05$ was statistically significant. 
A

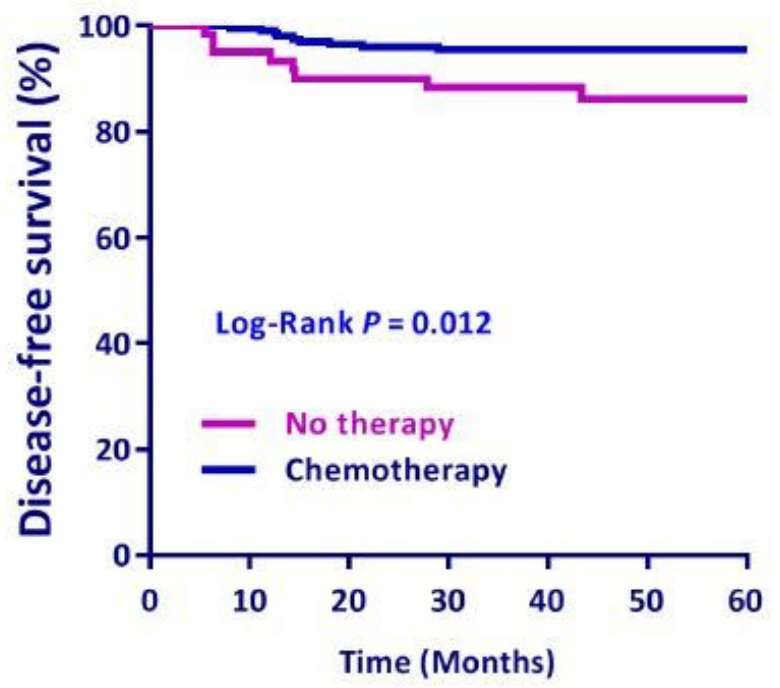

B

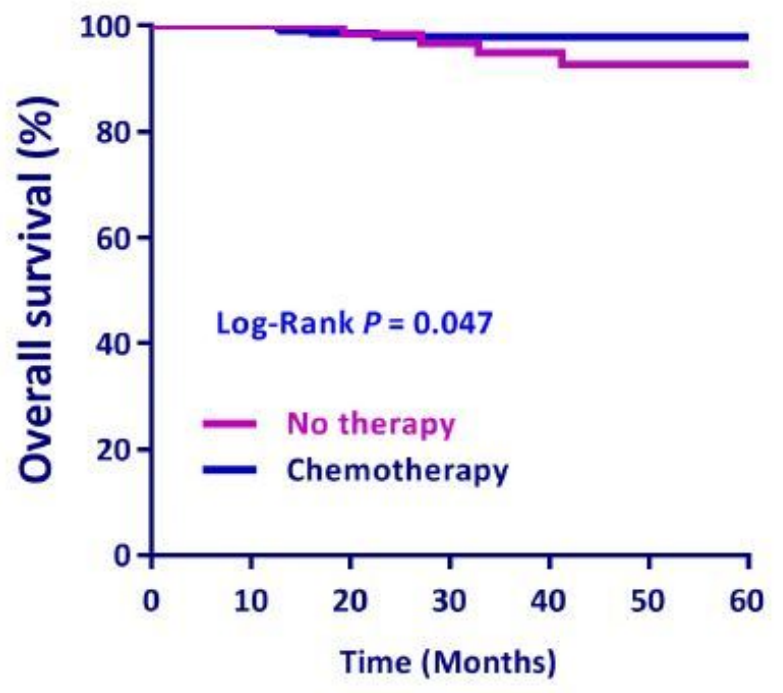

Figure 1

(A) Disease-free survival (DFS) and (B) overall survival (OS) by postoperative chemotherapy. Differences in DFS and OS by treatment were evaluated using the log-rank test. $\mathrm{P}<0.05$ was statistically significant.

A

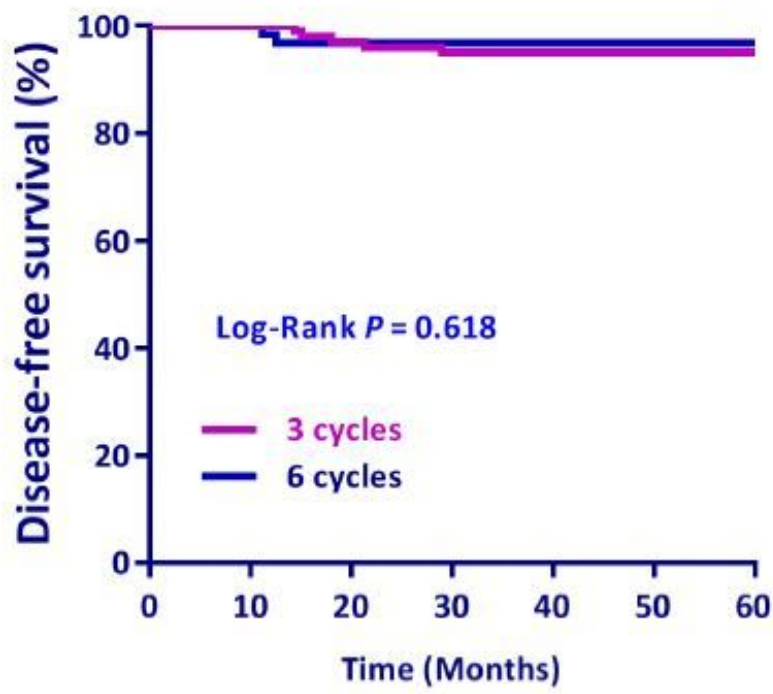

B

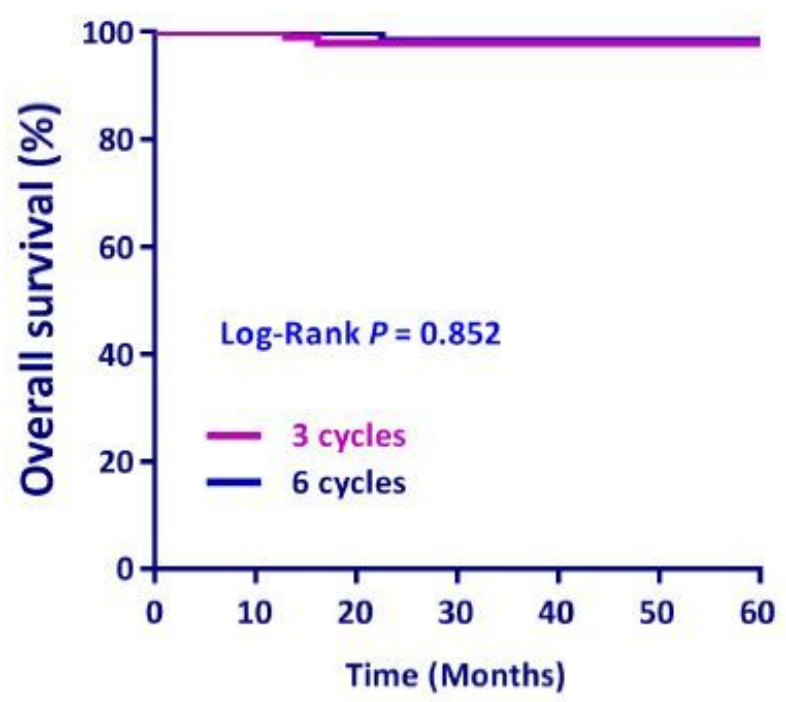

Figure 2

(A) Disease-free survival (DFS) and (B) overall survival (OS) by postoperative chemotherapeutic cycle. Differences in DFS and OS by treatment were evaluated using the log-rank test. 